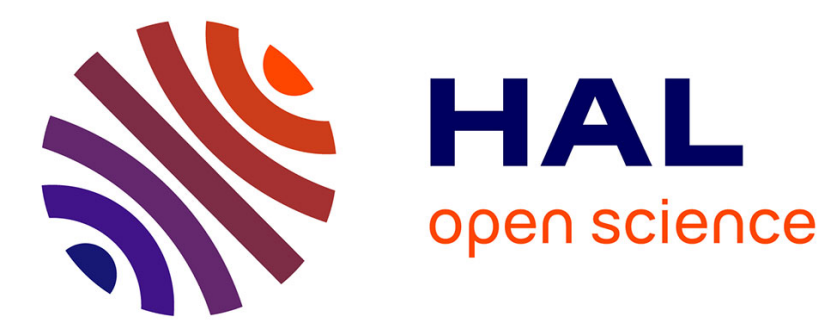

\title{
Improved nonlinear slot waveguides using dielectric buffer layers: properties of TM waves
}

\author{
Mahmoud M. R. Elsawy, Gilles Renversez
}

\section{To cite this version:}

Mahmoud M. R. Elsawy, Gilles Renversez. Improved nonlinear slot waveguides using dielectric buffer layers: properties of TM waves. Optics Letters, 2016, 41 (7), pp.1542-1545. 10.1364/OL.41.001542 . hal-01280607

\section{HAL Id: hal-01280607 \\ https://hal.science/hal-01280607}

Submitted on 29 Mar 2016

HAL is a multi-disciplinary open access archive for the deposit and dissemination of scientific research documents, whether they are published or not. The documents may come from teaching and research institutions in France or abroad, or from public or private research centers.
L'archive ouverte pluridisciplinaire HAL, est destinée au dépôt et à la diffusion de documents scientifiques de niveau recherche, publiés ou non, émanant des établissements d'enseignement et de recherche français ou étrangers, des laboratoires publics ou privés.

\section{(ㅇ)(1) $\$$}

Distributed under a Creative Commons Attribution - NonCommercial - NoDerivatives $\mid 4.0$ 


\title{
Improved nonlinear slot waveguides using dielectric buffer layers: properties of TM waves
}

\author{
Mahmoud M. R. Elsawy ${ }^{1}$ And Gilles Renversez ${ }^{1, *}$ \\ ${ }^{1}$ Aix-Marseille Université, CNRS, Ecole Centrale Marseille, Institut Fresnel UMR 7249, 13013 Marseille, France \\ *Corresponding author: gilles.renversez@univ-amu.fr
}

Compiled March 29, 2016

We propose an improved version of the symmetric metal slot waveguides with a Kerr-type nonlinear dielectric core adding linear dielectric buffer layers between the metal regions and the core. Using a finite element method to compute the stationary nonlinear modes, we provide the full phase diagrams of its main TM modes as a function of total power, buffer layer and core thicknesses, that are more complex than the ones of the simple nonlinear metal slot. We show that these modes can exhibit spatial transitions towards specific modes of the new structure as a function of power. We also demonstrate that, for the main modes, the losses are reduced compared to the previous structures, and that they can now decrease with power. Finally, we describe the stability properties of the main stationary solutions using nonlinear FDTD simulations.

OCIS codes: $\quad 190.6135,240.6680,230.7400,190.3270$

http://dx.doi.org/10.1364/ol.XX.XXXXXX

Nonlinear plasmonic slot waveguides (NPSWs) have drawn attention in the last decade due to the strong light confinement in the nonlinear dielectric core ensured by the surrounding metal regions, and to their peculiar nonlinear effects [1-6]. Several applications have already been proposed for NPSWs $[7,8]$. Nevertheless, the experimental observation of plasmon-soliton waves in these NPSWs is still lacking even if linear slot waveguides have already been fabricated [9]. Similarly to the case of the single nonlinear dielectric/metal interface structures [10, 11], the modes already studied in the simple NPSWs suffer from high losses that seriously limit the propagation length of the waves. In the present study, we propose and study an improved structure in which buffer linear dielectric layers are added between the nonlinear dielectric core and the two semi-infinite metal regions. The article is organized as follows. First, we describe the model and numerical method we use to study the stationary nonlinear waves in the improved NPSW we propose. Secondly, we describe the linear solutions of the new structures in order to classify the nonlinear solutions. Thirdly, we give the properties of the nonlinear stationary solutions and show that, for some linear parameter configurations, new modal spatial transitions as a function of power occur compared to the simple
NPSW case. We provide full phase diagrams for the improved NPSW as a function of total power, buffer layer and core thicknesses. We also prove that the added buffer dielectric layers are able to reduce losses and allow them to decrease with power in most of the cases. Finally, using the FDTD method we study the stability properties of the main nonlinear solutions.

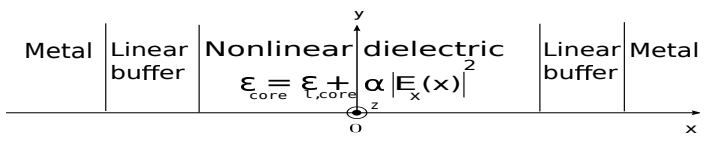

Fig. 1. Symmetric improved NPSW geometry with the supplementary linear dielectric buffer layers between the nonlinear dielectric core and the two semi-infinite metal regions.

The improved NPSW we study is depicted in Fig. 1. Compared to already studied simple NPSW made of a nonlinear dielectric core surrounded by two semi-infinite metal regions $[1,2,6]$, the improved structure contains additional linear dielectric layers between the core and the metal regions. In this study, we will consider only symmetric structures even if asymmetric simple NPSWs have already been considered [12]. $d_{\text {core }}$ is the core thickness, and $d_{b u f}$ is the dielectric buffer thickness of each of the supplementary layers with their permittivities denoted by $\varepsilon_{b u f}$. We will only consider monochromatic transverse magnetic (TM) waves propagating along the $z$ direction in a symmetric one-dimensional NPSW. All field components evolve proportionally to $\exp \left[i\left(k_{0} n_{\text {eff }} z-\omega t\right)\right]$ with $k_{0}=\omega / c$, where $c$ denotes the speed of light in vacuum, $\omega$ is the angular frequency, and $n_{e f f}$ denotes the effective mode index. The nonlinear Kerr-type dielectric is isotropic with $\varepsilon_{\text {core }}=\varepsilon_{l, \text { core }}+\alpha\left|E_{x}\right|^{2}$ ( $\varepsilon_{l, \text { core }}$ being the linear part of the core permittivity, $E_{x}$ being the $x$ component of the electric field), and with $\alpha>0$ (focusing nonlinearity). This approximation of the Kerr nonlinearity, taking into account only the transverse component of the electric field has already been used in models of the NPSWs [6, 12]. This approximation allows us to use the fixed power algorithm in the finite element method (FEM) to compute the nonlinear stationary solutions and their nonlinear dispersion curves [13-16].

In the core, the approximated relation between the nonlinear parameter $\alpha$ and the coefficient $n_{2}$, that appears in the definition of an intensity dependent refractive index $\Re e(n)=$ $\Re e\left(\varepsilon_{l, \text { core }}\right)^{1 / 2}+n_{2} I$, is $\alpha=\varepsilon_{0} c \Re e\left(\varepsilon_{l, \text { core }}\right) n_{2}$ where the intensity is defined as $I=\varepsilon_{0} c\left|\varepsilon_{\text {core }}\right|\left|E_{x}\right|^{2} /\left(2 n_{e f f}\right)$, and the vacuum per- 
mittivity is denoted by $\varepsilon_{0}$. To compute the linear and nonlinear modes we use our custom-built FEM developed to study scalar and vector models for waveguides including nonlinear ones with Kerr regions like microstructured optical fibers and NPSWs [15-17]. In this study, $\varepsilon_{l, \text { core }}=3.46^{2}+i 10^{-4}$ and $n_{2}=1.10^{-17} \mathrm{~m}^{2} / \mathrm{W}$ corresponding to amorphous hydrogenated silicon [6], and $\varepsilon_{m e t}=-90+i 10$ corresponding to gold at 1.55 $\mu \mathrm{m}$, wavelength for which all the simulations are done. Losses will be estimated using the method based on the field profiles and permittivity imaginary parts described in $[3,10,12,18]$. We stress that used parameter values can affect the given critical values but would not affect its main conclusions.

We start by the study of the linear case as it is well known that this is a crucial step in the investigation of nonlinear waveguides [19]. Figure 2(a) provides the linear dispersion curves for symmetric improved NPSWs as a function of the thickness of supplementary buffer dielectric layers. The horizontal line represents the linear part of core refractive index $\Re e\left(\varepsilon_{l, \text { core }}\right)$. It splits the different dispersion curves in two parts with distinct types of field profiles. Below this line, the mode is mostly core localized and its $H_{y}$ component follows the cosine profile in the linear high-index core with plasmonic tails in the metal regions as it can be seen in Fig. 2(b). Above this line, the mode is of plasmonic type, as in the simple NPSW, as shown in Fig. 2(b). It is important to notice that the first type of mode is not found in the simple NPSW as a linear mode whatever the used optogeometric parameters $[12,20]$,but a corresponding mode can be observed in structures with a semi-infinite nonlinear region and a metal one separated by a dielectric buffer layer [5].

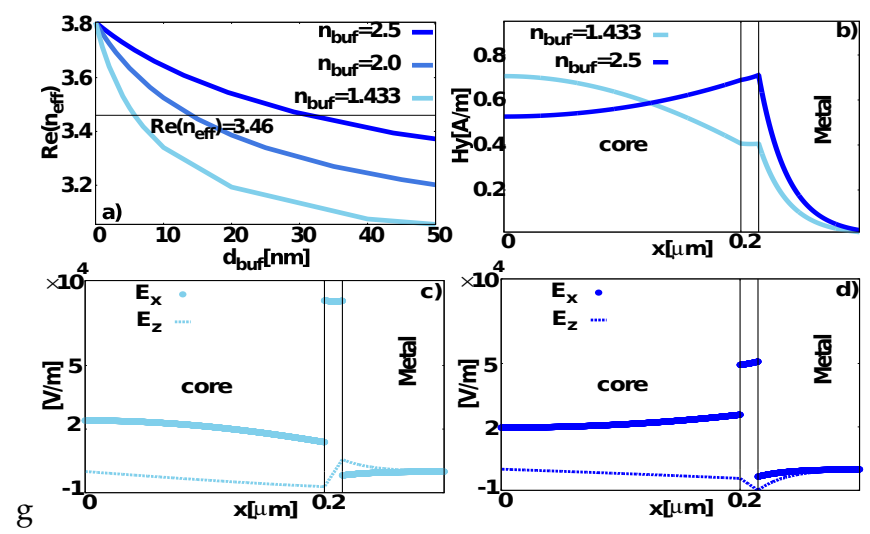

Fig. 2. (a) Linear dispersion curves of the fundamental symmetric mode for the improved NPSW with $d_{\text {core }}=400 \mathrm{~nm}$ as a function of $d_{b u f}$ for three buffer layer refractive indices $n_{b u f}$. Typical magnetic field symmetric components $H_{y}(x)$ for $d_{b u f}=15 \mathrm{~nm}$ : (b) with a cosine-type profile for $\varepsilon_{b u f}=1.443^{2}$ such that $\Re e\left(n_{e f f}\right)<\Re e\left(\varepsilon_{l, c o r e}\right)^{1 / 2}$ and with a plasmonic-type profile $\varepsilon_{b u f}=2.5^{2}$ such that $\Re e\left(n_{e f f}\right)>\Re e\left(\varepsilon_{l, \text { core }}\right)^{1 / 2}$. (c) associated $E_{x}(x)$ and $E_{z}(x)$ components for $\varepsilon_{b u f}=1.443^{2}$. (d) associated $E_{x}(x)$ and $E_{z}(x)$ components for $\varepsilon_{b u f}=2.5^{2}$.

For the nonlinear study, we start with the case such that the effective index of the symmetric linear mode is below the core linear refractive index. The real parts of effective indices of the first three main modes: symmetric, asymmetric and antisymmetric denoted respectively by S0-solI, AS1-solI, and AN0-solI, are shown in Fig. 3(a) while their imaginary parts and losses are shown in Fig. 3(b). In the two previous figures, the results for a simple NPSW made from the improved NPSW by removing the buffer dielectric layers are also shown in grey for comparison (symmetric S0-plas, asymmetric AS1-plas, antisymmetric AN0plas). The mode notation used in our study has been chosen to extend coherently the one already introduced for the simple NPSW $[12,20]$. As it can be seen, in the improved NPSW, the bifurcation of the asymmetric mode from the symmetric mode and the relative positions of the three main modes are preserved. The difference between the symmetric mode curve and the asymmetric mode curve is smaller in this new structure. The reason is that, in this structure, the symmetric mode profile looks like a symmetric hyperbolic secant spatial soliton and its associated asymmetric mode is simply shifted off the slot center. We also note that losses are at least three times smaller than the ones of the simple NPSW (see Fig. 3(b)).
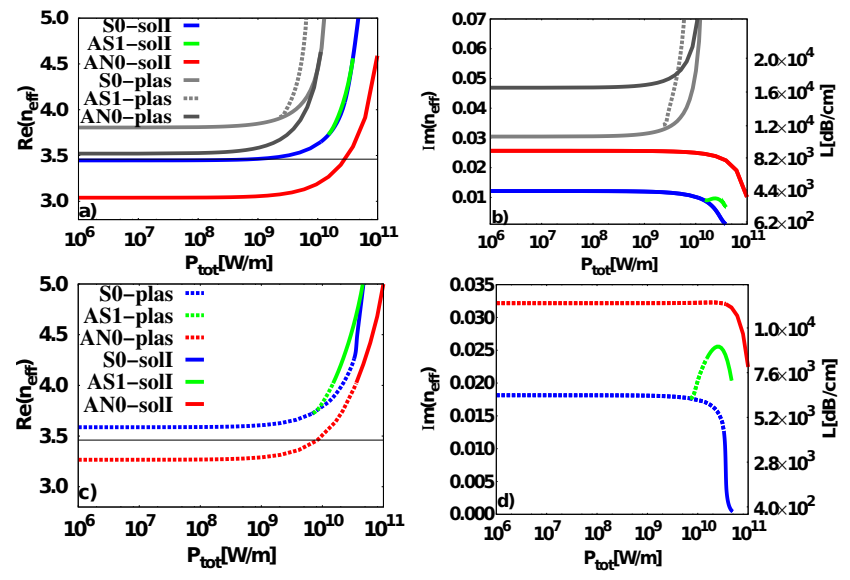

Fig. 3. Nonlinear dispersion curves for the simple NPSW (grey curves) and for the improved one (color curves). The blue, green, and red colors indicate the symmetric, asymmetric and antisymmetric nonlinear modes respectively. $d_{\text {core }}=400 \mathrm{~nm}$, $d_{b u f}=15 \mathrm{~nm}$. For $\varepsilon_{b u f}=2^{2}$ : (a) for $\Re e\left(n_{e f f}\right)$ and (b) for $\Im m\left(n_{e f f}\right)$ and the associated losses. For $\varepsilon_{b u f}=2.5^{2}$ : (c) for $\Re e\left(n_{e f f}\right)$ and $(\mathrm{d})$ for $\Im m\left(n_{\text {eff }}\right)$ and the associated losses.

We now study the nonlinear dispersion curves of the improved NPSW such that the effective index of the symmetric linear mode is above the core linear refractive index. The corresponding curves are given in Figs. 3(c) and (d). The global shape of these dispersion curves is similar to the ones studied for the three main modes of the simple NPSW but the mode field profiles now exhibit a spatial transition from a plasmonic type profile to a solitonic type one: S0-plas to S0-solI for the symmetric mode, AS1-plas to AS1-soll for the asymmetric one, and AN0-plas to AN0-solI for the antisymmetric one (see field profiles in Fig. 4). Similar transitions have already been observed in structures with a semi-infinite nonlinear region [10, 21, 22], but not in NPSWs [16]. Furthermore, at low powers in the improved NPSWs, the losses are reduced, at least, by a factor two compared to the associated simple NPSW, and they are now decreasing with the power for the main symmetric and antisymmetric modes leading to low loss solutions at high powers (see Fig. 3(d)). For the asymmetric mode, loss first increases with power and then decreases. This loss increase is linked to the fact that, in this case, the field first tends to increase near one of the metal interface, increasing the fraction of field in a highly lossy region. Then, the mode moves gradually from a plasmonic type to a solitonic type asymmetric profile, and when the soliton peak 
is far enough from the metal interface losses start to decrease.

Figure 4 shows the profiles of the first three main modes as a function of power. The symmetric solitonic mode S0-solI (see solid curves in Figs. 4(a) and 4(d)) looks like the higher order purely nonlinear symmetric mode denoted SI we have found in the simple NPSW but with different boundary conditions at the core interface due to the opposite sign of the real part of the permittivity in the outer regions [6, 20]. In Fig. 5, we show
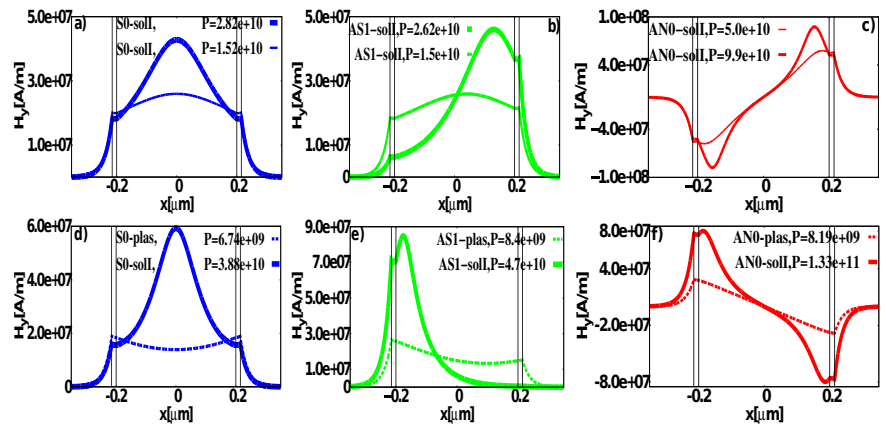

Fig. 4. Field profile $H_{y}(x)$ for symmetric (blue curves), asymmetric (green curves), and antisymmetric (red curves) modes with $\varepsilon_{b u f}=2^{2}$ (top), and with $\varepsilon_{b u f}=2.5^{2}$ (bottom) before (dashed line) and after (solid line) the spatial modal transition induced by the power increase. The power is given in $\mathrm{W} / \mathrm{m}$.

the influence of the buffer thickness $d_{b u f}$ on the losses of the symmetric mode at low power. As expected losses decrease with $d_{b u f}$, and interestingly, it exists a critical $d_{b u f}$ value which separates the plasmonic type solutions (dashed curves) from the solitonic type solutions (solid lines) as it can be inferred from the linear dispersion curves given in Fig. 2(a). Using numeri-
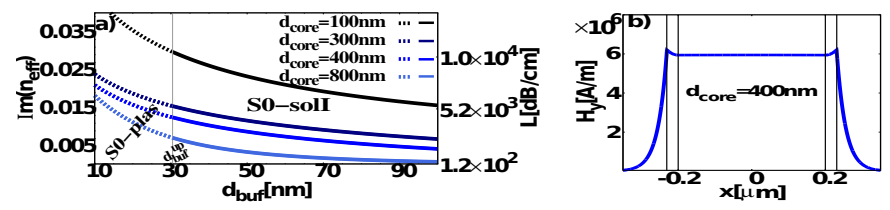

Fig. 5. (a) Losses of the symmetric mode for a total power $P_{\text {tot }}=1.010^{9} \mathrm{~W} / \mathrm{m}$ as a function of the buffer thickness $d_{b u f}$ for four different core thicknesses with $\varepsilon_{b u f}=2.5^{2}$. The dashed lines are for plasmon type solutions and solid ones are for soliton type ones. (b) Flat magnetic field profile obtained for the upper critical value $d_{b u f}=d_{b u f}^{u p}=30.8 \mathrm{~nm}$.

cal simulations providing both the dispersion curves and the associated field profiles like the ones shown above, we obtain the phase diagram of the improved NPSW for the main modes. Such diagram is shown in Fig. 6 where the existence and the type of the first symmetric and asymmetric modes are given. The buffer thickness $d_{b u f}$ and the total power $P_{\text {tot }}$ are used as parameters. The upper critical value $d_{b u f}^{u p}$ is $30.8 \mathrm{~nm}$. Above this critical value, one has $\Re e\left(n_{e f f}\right)<\Re e\left(n_{l, \text { core }}\right)$ and the nonlinear symmetric mode emerges from the cosine linear solution (see Fig. 2(b)). It is of S0-soll type (symmetric soliton-type with a single peak, see solid profile in Fig. 4(a)). For the critical value $d_{b u f}^{u p}$, the black circle in Fig. 6 corresponds to the limit case where the $H_{y}$ field profile is still flat (see Fig. 5(b) for an example of such profile), for higher powers a core peak emerges. For $d<d_{b u f}^{u p}$, one has $\Re e\left(n_{e f f}\right)>\Re e\left(n_{l, c o r e}\right)$ and the first symmetric mode of the NPSW is of plasmonic type (see dashed curve in Fig. 4(d)). Below this critical value for $d_{b u f}$, and under the brown transition curve in Fig. 6, one has the S0-plas region, and above this curve the first symmetric mode is now of S0-soll type (see solid profile in Fig. 4(d)). Along the brown curve in the phase diagram, the $H_{y}$ field profile is flat. From $d_{b u f}^{\text {down }}=14.5 \mathrm{~nm}$, the lower critical $d_{b u f}$ value, a new type of symmetric nonlinear solution emerges, at high powers, from the S0-plas mode; it is composed of two solitons located in the core near each interfaces (see its symmetric field profile in inset in Fig. 7). We denote it S0-solII. The transition curve between the S0-plas region and the S0-solII region is shown in cyan in the phase diagram. The limit curve above which the S0-solI mode appears is the pink one in Fig. 6. It is worth mentioning that in the region above the cyan transition curve and below the pink limit curve, only the S0-solII mode exists. In Fig. 6, we also provide the behaviour of the first asymmetric mode (green curves). The dashed green curve is the limit curve of the asymmetric plasmonic mode AS1-plas (see Fig. 4(e)): below the curve, it does not exist. It emerges at low powers from the symmetric one, S0-plas (see Fig. 4(d)). At higher powers (see the solid green line), it moves to an asymmetric mode of solitonic type AS1-solI (a peak is located in the core near one of its interface, see solid profile in Fig. 4(e)). Above $d_{b u f} \simeq 24 \mathrm{~nm}$, only this AS1-solI mode exists and it emerges from the S0-solI symmetric mode as expected (see Figs. 4(d) and (e)).

The permittivities being fixed, we numerically studied the influence of the core thickness $d_{\text {core }}$ on the critical values $d_{b u f}^{\text {down }}$, and $d_{b u f}^{u p}$ (see Fig. 5), we found that $d_{b u f}^{u p}$ does not change with $d_{\text {core }}$ for $d_{\text {core }} \geq 10 \mathrm{~nm}$, while $d_{\text {buf }}^{\text {down }}$ increases with $d_{\text {core }}$ (data not shown), such that they coincide when the core thickness reaches the critical value $d_{\text {core }}^{\lambda} \simeq 1550$ nm (one wavelength), above this core thickness, the critical value $d_{b u f}^{\text {down }}$ will no longer change with $d_{\text {core }}$. Actually, it is possible to get an analytical approximation for $d_{b u f}^{u p}$ considering a buffer layer sandwiched between semi-infinite metal and high-index dielectric regions, one gets, when $\left|\Re e\left(\varepsilon_{\text {met }}\right)\right| \gg\left|\varepsilon_{l, \text { core }}\right|, d_{b u f}^{u p}=$ $\left(\lambda \varepsilon_{\text {buf }}\right) /\left(2 \pi\left|\Re e\left(\varepsilon_{\text {met }}\right)\right|^{1 / 2}\left(\Re e\left(\varepsilon_{l, \text { core }}\right)-\Re e\left(\varepsilon_{\text {buf }}\right)\right)\right) \simeq 28.4 \mathrm{~nm}$. This simple approximated value agrees well with the simulations results $(30.8 \mathrm{~nm}$ ) while the full formula (not shown) for $d_{b u f}^{u p}$ gives the better approximation $31.1 \mathrm{~nm}$. Since at $d_{b u f}^{u p}$, the mode is flat in the nonlinear core (see Fig. 5(b)) as in the approximated model, it can be understood why both formula, that do not depend on $d_{\text {core }}$, provide a good approximation of $d_{b u f}^{u p}$.

In Fig. 7, we provide the phase diagram of the improved NPSW using $d_{\text {core }}$ and $P_{\text {tot }}$ as parameters. The meaning of the curves are the same as in Fig. 6. We found that the threshold for the Hopf bifurcation, from which the asymmetric mode emerges, strongly decreases with the core thickness as it was already found in the simple NPSW $[6,20]$. One clearly sees that there is a critical value, denoted by $d_{\text {core }}^{\text {crit }}$ around $430 \mathrm{~nm}$ for $d_{\text {buf }}=15 \mathrm{~nm}$ such that below it, only the S0-plas and the S0-solI modes exist (see Fig. $4(\mathrm{~d})$ ). This low $d_{\text {core }}$ region is split in two parts by the transition curve (brown one): below it, only the S0-plas exists while above only the $\mathrm{S} 0$-solI exists as fundamental symmetric mode.

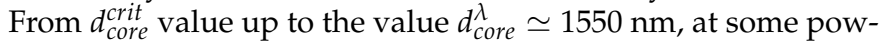
ers the S0-plas mode move to the S0-solII mode (cyan curve) and at even higher powers the S0-solI mode emerges (pink curve). For $d_{\text {core }}$ above $1550 \mathrm{~nm}$, the emergence of the S0-solI mode appears at powers lower than the transition between the S0-plas mode and the S0-solII one. 


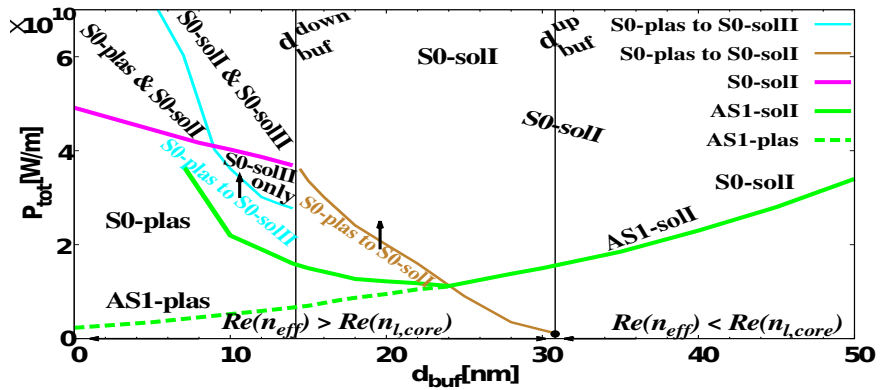

Fig. 6. Phase diagram of the improved NPSW as a function of dielectric buffer thickness $d_{b u f}$ and of the total power $P_{t o t}$ for $d_{\text {core }}=400 \mathrm{~nm}$ and $\varepsilon_{\text {buf }}=2.5^{2}$. The green curves refer to the first asymmetric modes. Arrows indicate the corresponding mode transition across the associated curve (thin one).

The green AS1-plas and the AS1-soll dispersion curves (see mode profiles in Fig. 4(e)) shown in Fig. 7 merge when they cross the brown curve associated with the transition from the S0-plas mode to the S0-solI one for increasing power. A similar phenomenom was already visible in Fig. 6. After this merging, above the brown S0-plas/S0-solI transition curve, the AS1-plas mode does not exist anymore. In a previous work, using two dif-

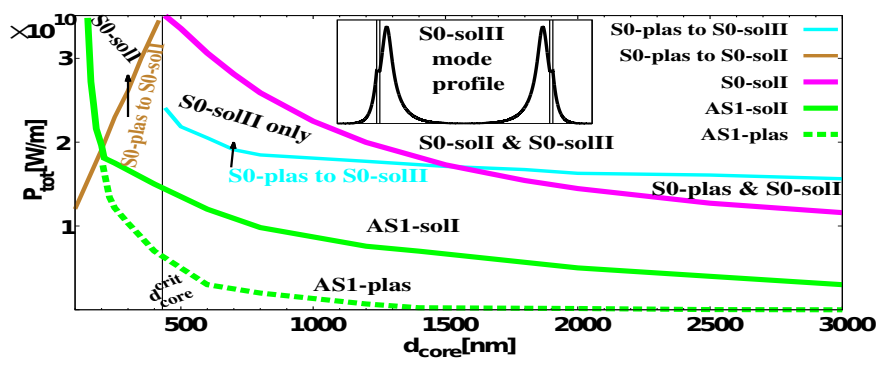

Fig. 7. Phase diagram of the improved NPSW as a function of the nonlinear core thickness $d_{\text {core }}$ and of $P_{t o t}$, for $d_{b u f}=15 \mathrm{~nm}$ and $\varepsilon_{b u f}=2.5^{2}$. The green curves refer to the first asymmetric mode. Inset: $H_{y}(x)$ field profile for the S0-solII mode.

ferent numerical methods including the FDTD, we have already shown that the results about the stability of the main symmetric and asymmetric modes derived in reference [23] from theoretical arguments in the framework of the weak guidance approximation in nonlinear waveguides are still valid, at least numerically, in simple NPSWs except just around the bifurcation point where subtle behaviours occur [20]. Here, we have again used the nonlinear capabilities of the FDTD method implemented in the MEEP software [24]. The metal permittivity is described by a Drude model to obtain the fixed negative value used at the studied wavelength in the stationnary study. As it can be seen in Fig. 8, for the improved NPSW, the symmetric mode S0-soll is stable at low power. The S0-plas mode (plasmonic type) is also stable as expected from the results in [20] for the simple NPSW. The asymmetric mode AS1-solI is also stable for the tested powers above the bifurcation threshold (data not shown). Due to the losses of the metal, and as pointed out in [20, 25], the temporal nonlinear solutions are actually not self-coherent [19] as it can be seen in Fig. 8 with the decrease of the field intensity along the propagation.

We have proposed an improved nonlinear plasmonic slot waveguide adding supplementary dielectric buffer layers be-

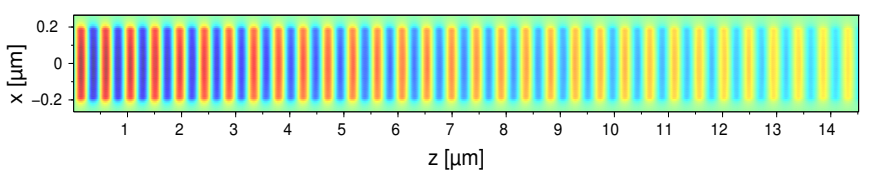

Fig. 8. Evolution during the propagation of the $H_{y}$ field profile of the S0-soll symmetric solitonic type mode at a power such that $\langle\Delta n\rangle=0.043$ for $d_{\text {core }}=300 \mathrm{~nm}, d_{\text {buf }}=40 \mathrm{~nm}, \varepsilon_{b u f}=2.5^{2}$, other parameters as in the text. $\langle\Delta n\rangle$ is the spatial average along the core of the nonlinear refractive index change $[6,20]$.

tween the nonlinear core and the metal regions. We have provided a complete description of its main nonlinear TM modes. We have shown that this structure allows a decrease of the losses compared to the simple nonlinear slot waveguide, and that its main modes exhibit, for appropriate opto-geometric parameters, spatial transitions that can be controlled by the power. The stability of the main modes has also been demonstrated numerically.

\section{REFERENCES}

1. E. Feigenbaum and M. Orenstein, Opt. Lett. 32, 674-676 (2007).

2. A. R. Davoyan, I. V. Shadrivov, and Y. S. Kivshar, Opt. Express 16, 21209-21214 (2008).

3. A. R. Davoyan, I. V. Shadrivov, and Y. S. Kivshar, Opt. Express 17, 21732-21737 (2009).

4. I. D. Rukhlenko, A. Pannipipitiya, M. Premaratne, and G. Agrawal, Phys. Rev. B 84, 113409 (2011).

5. A. Ferrando, C. Milián, and D. V. Skryabin, J. Opt. Soc. Am. B 30, 2507-2522 (2013).

6. W. Walasik, A. Rodriguez, and G. Renversez, Plasmonics 10, 33-38 (2015).

7. J. R. Salgueiro and Y. S. Kivshar, Appl. Phys. Lett. 97, 081196 (2010).

8. N. Nozhat and N. Granpayeh, Opt. Commun. 285, 1555-1559 (2012).

9. Z. Han, A. Y. Elezzabi, and V. Van, Opt. Lett. 35, 502-504 (2010).

10. G. I. Stegeman and C. T. Seaton and J. Ariyasu and R. F. Wallis and A. A. Maradudin, J. Appl. Phys. 58, 2453-2459 (1985).

11. W. Walasik, V. Nazabal, M. Chauvet, Y. Kartashov, and G. Renversez, Opt. Lett. 37, 4579-4581 (2012).

12. W. Walasik and G. Renversez, Phys. Rev. A 93, 013825 (2016).

13. B. M. A. Rahman, J. R. Souza, and J. B. Davies, IEEE Photon. Technol. Lett. 2, 265-267 (1990).

14. A. Ferrando, M. Zacarés, P. F. de Cordoba, D. Binosi, and J. A. Monsoriu, Optics Express 11, 452-459 (2003).

15. F. Drouart, G. Renversez, A. Nicolet, and C. Geuzaine, J. Opt. A: Pure Appl. Opt. 10, 125101 (2008).

16. W. Walasik, G. Renversez, and Y. V. Kartashov, Phys. Rev. A 89, 023816 (2014).

17. F. Zolla, G. Renversez, A. Nicolet, B. Kuhlmey, S. Guenneau, D. Felbacq, A. Argyros, and S. Leon-Saval, Foundations of Photonic Crystal Fibres (Imperial College Press, London, 2012), 2nd ed.

18. A. W. Snyder and J. D. Love, Optical Waveguide Theory (Chapman and Hall, London, 1983).

19. A. W. Snyder, D. J. Mitchell, and L. Poladian, J. Opt. Soc. Am. B 8, 1618-1620 (1991)

20. W. Walasik, G. Renversez, and F. Ye, Phys. Rev. A 93, 013826 (2016).

21. K. Y. Bliokh, Y. P. Bliokh, and A. Ferrando, Phys. Rev. A 79, 041803 (2009).

22. C. Miliàn, D. E. Ceballos-Herrera, D. V. Skryabin, and A. Ferrando, Opt. Lett. 37, 4221-4223 (2012).

23. D. J. Mitchell and A. W. Snyder, J. Opt. Soc. Am. B 10, 1572-1580 (1993).

24. A. F. Oskooi, D. Roundy, M. Ibanescu, P. Bermel, J. D. Joannopoulos, and S. G. Johnson, Comput. Phys. Commun. 181, 687-702 (2010).

25. J. R. Salgueiro and Y. S. Kivshar, J. of Optics 16, 114007 (2014). 


\section{REFERENCES}

1. E. Feigenbaum and M. Orenstein, "Plasmon-soliton," Opt. Lett. 32, 674-676 (2007).

2. A. R. Davoyan, I. V. Shadrivov, and Y. S. Kivshar, "Nonlinear plasmonic slot waveguide," Opt. Express 16, 21209-21214 (2008).

3. A. R. Davoyan, I. V. Shadrivov, and Y. S. Kivshar, "Self-focusing and spatial plasmon-polariton solitons," Opt. Express 17, 21732-21737 (2009).

4. I. D. Rukhlenko, A. Pannipipitiya, M. Premaratne, and G. Agrawal, "Exact dispersion relation for nonlinear plasmonic waveguides," Phys. Rev. B 84, 113409 (2011).

5. A. Ferrando, C. Milián, and D. V. Skryabin, "Variational theory of soliplasmon resonances," J. Opt. Soc. Am. B 30, 2507-2522 (2013).

6. W. Walasik, A. Rodriguez, and G. Renversez, "Symmetric plasmonic slot waveguides with a nonlinear dielectric core: Bifurcations, size effects, and higher order modes," Plasmonics 10, 33-38 (2015).

7. J. R. Salgueiro and Y. S. Kivshar, Nonlinear plasmonic directional couplers, Appl. Phys. Lett. 97, 081196 (2010).

8. N. Nozhat and N. Granpayeh, "Switching power reduction in the ultracompact Kerr nonlinear plasmonic directional coupler," Opt. Commun. 285, 1555-1559 (2012).

9. Z. Han, A. Y. Elezzabi, and V. Van, "Experimental realization of subwavelength plasmonic slot waveguides on a silicon platform," Opt. Lett. 35, 502-504 (2010).

10. G. I. Stegeman and C. T. Seaton and J. Ariyasu and R. F. Wallis and A. A. Maradudin, J. Appl. Phys. 58, 2453-2459 (1985).

11. W. Walasik, V. Nazabal, M. Chauvet, Y. Kartashov, and G. Renversez, "Low-power plasmon-soliton in realistic nonlinear planar structures," Opt. Lett. 37, 4579-4581 (2012).

12. W. Walasik and G. Renversez, "Plasmon-soliton waves in planar slot waveguides. I. modeling," Phys. Rev. A 93, 013825 (2016).

13. B. M. A. Rahman, J. R. Souza, and J. B. Davies, "Numerical analysis of nonlinear bistable optical waveguides," IEEE Photon. Technol. Lett. 2, 265-267 (1990).

14. A. Ferrando, M. Zacarés, P. F. de Cordoba, D. Binosi, and J. A. Monsoriu, "Spatial soliton formation in photonic crystal fibers," Optics Express 11, 452-459 (2003).

15. F. Drouart, G. Renversez, A. Nicolet, and C. Geuzaine, "Spatial Kerr solitons in optical fibres of finite size cross section: beyond the Townes soliton," J. Opt. A: Pure Appl. Opt. 10, 125101 (2008).

16. W. Walasik, G. Renversez, and Y. V. Kartashov, "Stationary plasmonsoliton waves in metal-dielectric nonlinear planar structures: modeling and properties," Phys. Rev. A 89, 023816 (2014).

17. F. Zolla, G. Renversez, A. Nicolet, B. Kuhlmey, S. Guenneau, D. Felbacq, A. Argyros, and S. Leon-Saval, Foundations of Photonic Crystal Fibres (Imperial College Press, London, 2012), 2nd ed.

18. A. W. Snyder and J. D. Love, Optical Waveguide Theory (Chapman and Hall, London, 1983).

19. A. W. Snyder, D. J. Mitchell, and L. Poladian, "Linear approach for approximating spatial solitons and nonlinear guided modes," J. Opt. Soc. Am. B 8, 1618-1620 (1991).

20. W. Walasik, G. Renversez, and F. Ye, "Plasmon-soliton waves in planar slot waveguides. II. results for stationary waves and stability analysis," Phys. Rev. A 93, 013826 (2016).

21. K. Y. Bliokh, Y. P. Bliokh, and A. Ferrando, "Resonant plasmon-soliton interaction," Phys. Rev. A 79, 041803 (2009).

22. C. Miliàn, D. E. Ceballos-Herrera, D. V. Skryabin, and A. Ferrando, "Soliton-plasmon resonances as Maxwell nonlinear bound states," Opt. Lett. 37, 4221-4223 (2012).

23. D. J. Mitchell and A. W. Snyder, "Stability of fundamental nonlinear guided waves," J. Opt. Soc. Am. B 10, 1572-1580 (1993).

24. A. F. Oskooi, D. Roundy, M. Ibanescu, P. Bermel, J. D. Joannopoulos, and S. G. Johnson, "MEEP: A flexible free-software package for electromagnetic simulations by the FDTD method," Comput. Phys. Commun. 181, 687-702 (2010).

25. J. R. Salgueiro and Y. S. Kivshar, "Complex modes in plasmonic nonlinear slot waveguides," J. of Optics 16, 114007 (2014). 